\title{
Time-resolved photoluminescence spectra of strong visible light-emitting SiC nanocrystalline films on Si deposited by electron-cyclotron-resonance chemical-vapor deposition
}

\author{
S. J. $X u^{a)}$ \\ Department of Physics, The University of Hong Kong, Hong Kong, China \\ M. B. Yu, Rusli, and S. F. Yoon \\ School of Electrical and Electronic Engineering, Nanyang Technological University, \\ Singapore 639798, Singapore \\ C. M. Che \\ Department of Chemistry, The University of Hong Kong, Hong Kong, China
}

(Received 18 November 1999; accepted for publication 13 March 2000)

\begin{abstract}
$\mathrm{SiC}$ nanocrystalline films on $\mathrm{Si}$ substrates deposited using advanced electron-cyclotron-resonance chemical-vapor deposition exhibit intense visible light emission at room temperature under laser excitation. Continuous-wave and time-resolved photoluminescence measurements for these $\mathrm{SiC}$ films were carried out at room temperature. The photon energy of the dominant emission peaks is higher than the band gap of cubic SiC. Room-temperature optical absorption measurements show a clear blueshift of the band gap of the samples with a decrease of the average size of the nanoclusters, indicating an expected quantum-confinement effect. However, the emission spectra are basically independent of the size. Temporal evolution of the dominant emissions exhibits double-exponential decay processes. Two distinct decay times of $\sim 200 \mathrm{ps}$ and $\sim 1 \mathrm{~ns}$ were identified, which are at least two orders of magnitude faster than that of the bound-exciton transitions in bulk $3 \mathrm{C}-\mathrm{SiC}$ at low temperature. Strong light emissions and short decay times strongly suggest that the radiative recombinations may be from some direct transitions such as self-trapped excitons on the surface of the nanoclusters. (C) 2000 American Institute of Physics. [S0003-6951(00)04618-0]
\end{abstract}

Silicon carbide $(\mathrm{SiC})$ is an "old" but important semiconductor material. In recent years, there is an overall renewed interest in this material due to development of new growth techniques. ${ }^{1-3} \mathrm{SiC}$ light-emitting diodes (LEDs) are one of its optoelectronic device applications. However, due to the intrinsic indirect-band-gap character, the quantum efficiency of the best SiC LED reported so far ${ }^{4}$ is still as low as about $10^{-4}$. Visible photoluminescence (PL) and electroluminescence (EL) of hydrogenated amorphous $\operatorname{SiC}(a-\mathrm{SiC}: \mathrm{H})$ films were observed in 1978 (Ref. 5) and 1983, ${ }^{6}$ respectively. Shortly thereafter, a so-called $a$-SiC:H thin-film LED made of $a-\mathrm{SiC}: \mathrm{H} p-i-n$ junctions was demonstrated. ${ }^{7}$ Although the brightness of this $a$-SiC:HLED is high enough to be observed by the eye, it is still too low for any practical application as a display panel. ${ }^{8}$ Microcrystalline $\mathrm{SiC}$ alloys deposited by electron-cyclotron-resonance chemical-vapor deposition (ECRCVD) were utilized as carrier injection layers to improve the performance of $a$-SiC:H LEDs. ${ }^{8}$ Recent research work ${ }^{9,10}$ in ECRCVD SiC coating technology has demonstrated the potential for fabricating high-quality $\mathrm{SiC}$ $\mathrm{x}$-ray mask membranes. Furthermore, the microstructure of $\mathrm{SiC}$ films on $\mathrm{Si}$ deposited by the ECRCVD technique was found to be an amorphous matrix embedded with nanosized crystalline particles. ${ }^{9,10}$ On the other hand, obviously inspired by the discovery of porous Si emitting strong luminescence, some groups reported PL and EL spectra of porous $\mathrm{SiC}$ fabricated using single-crystal $\mathrm{SiC}$ polytypes such as $3 \mathrm{C}$, $4 \mathrm{H}$, and $6 \mathrm{H} .{ }^{11-14}$ However, there has been little work on the

${ }^{a)}$ Electronic address: sjxu@hkucc.hku.hk luminescence properties of nanocrystalline $\mathrm{SiC}$ films deposited on Si using the ECRCVD technique. In this letter, we report the steady-state and transient luminescence characteristics of $\mathrm{SiC}$ nanocrystalline films grown on $\mathrm{Si}$ using ECRCVD. SiC films emit intense visible light at room temperature under excitation of a short-wavelength laser. The measured two luminescence decay time constants are $\sim 200$ ps and $1 \mathrm{~ns}$. The strongly enhanced luminescent efficiency may be due to direct optical transitions.

The $\mathrm{SiC}$ films investigated here were deposited on $\mathrm{Si}$ (100) substrates using the ECRCVD technique. Details of the growth will be published elsewhere. ${ }^{15}$ Here, we focus on the luminescent properties of two typical samples.

In the steady-state PL measurement, an argon-ion laser with a wavelength of $351 \mathrm{~nm}$ and output power of $5 \mathrm{~mW}$ was used as the excitation light source. The emission signal was dispersed in a double $0.25 \mathrm{~m}$ monochromoter and was detected by a photomultiplier tube using a standard lock-in amplification technique. For time-resolved PL measurements, the excitation light was $\sim 2$ ps pulses with a wavelength of $400 \mathrm{~nm}$ from frequency doubling of a $800 \mathrm{~nm}$ line of a mode-locked Ti:sapphire laser. The transient luminescence signal was dispersed in a $0.25 \mathrm{~m}$ monochromoter and was captured by a streak camera. The overall temporal resolution of the whole system is about $15 \mathrm{ps}$. Atomic-force microscopy (AFM) observation on the morphology of the samples was performed in a Digital Instruments NanoScope III with tapping mode.

Figures 1(a) and 1(b) show the AFM pictures of samples $\mathrm{D}$ and $\mathrm{E}$, respectively. Both samples were grown under the 

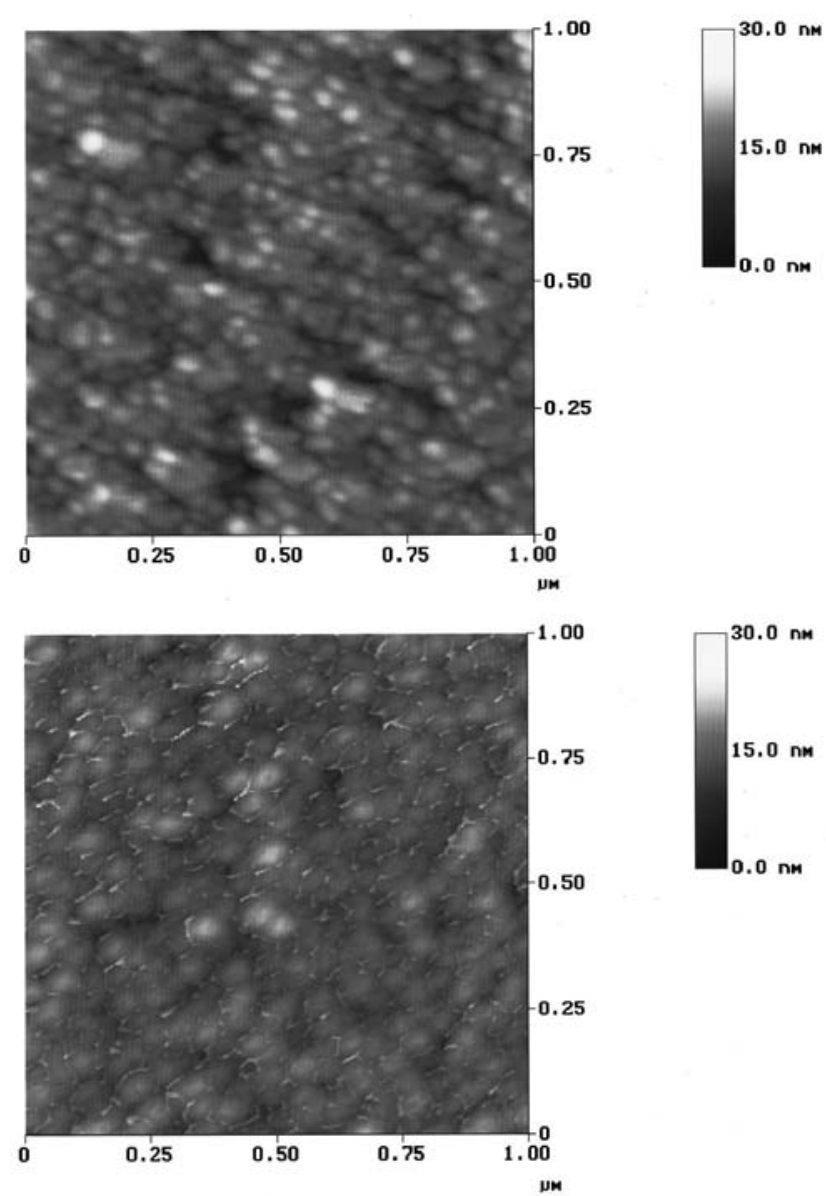

FIG. 1. AFM pictures of the SiC nanocrystal films of samples D (a) and E (b).

same conditions except for the gas flow ratio of the reaction gases. For sample D, the flow ratio of $\mathrm{H}_{2}: \mathrm{SiH}_{4}: \mathrm{CH}_{4}$ is $100: 10: 3$, while for sample $\mathrm{E}$, the flow ratio is 100:5:1. The nanosized clusters embedded in the films can be clearly seen in Fig. 1. Compared with that of sample D, the average diameter of the $\mathrm{SiC}$ clusters of sample $\mathrm{E}$ is larger. Moreover, the surface roughness of sample $\mathrm{E}$ is smaller than that of sample D. From the absorption spectra shown below, the size-dependent effect of the band gap of the $\mathrm{SiC}$ nanocrystals can be demonstrated. Figure 2 shows the room-temperature PL spectra and absorption spectra of samples D and E. We first discuss the absorption spectra of the $\mathrm{SiC}$ nanocrystals. Like porous $\mathrm{Si}$, the $\mathrm{SiC}$ nanocrystals exhibit unstructured optical absorption spectra due to the large distribution of the cluster size. The minimum indirect-band gap of cubic $\mathrm{SiC}$ (also called $3 \mathrm{C}-\mathrm{SiC}$ ) is the energy separation between $\Gamma_{15 \mathrm{~V}}$ and $X_{1 \mathrm{C}} \cdot{ }^{16}$ Its value is about $2.2 \mathrm{eV}$ at room temperature. ${ }^{17}$ Above this transition the absorption coefficient $\alpha$ should follow the well-known law of $\sqrt{\alpha(\hbar \omega)} \propto\left(\hbar \omega-E_{g 1}\right)$ for indirect-band-gap semiconductors. ${ }^{18}$ For an allowed direct transition, a photon energy dependence of the absorption coefficient should be expected as $\alpha(\hbar \omega)^{2} \propto\left(\hbar \omega-E_{g 2}\right) .{ }^{19}$ Following Ben-Chorin et al.'s work ${ }^{19}$ on Si nanocrystals, indirect band gaps extrapolated from measured absorption spectra are $\sim 2.32$ and $\sim 2.12 \mathrm{eV}$ for samples $\mathrm{D}$ and $\mathrm{E}$, respectively, which are close to the minimum indirect band gap of 3C-SiC. Possible direct band gaps of $\sim 3.5$ and $\sim 2.94 \mathrm{eV}$ are deduced for samples $\mathrm{D}$ and $\mathrm{E}$, respectively. Downloaded 03 Nov 2006 to 147.8.21.97. Redistribution subject to

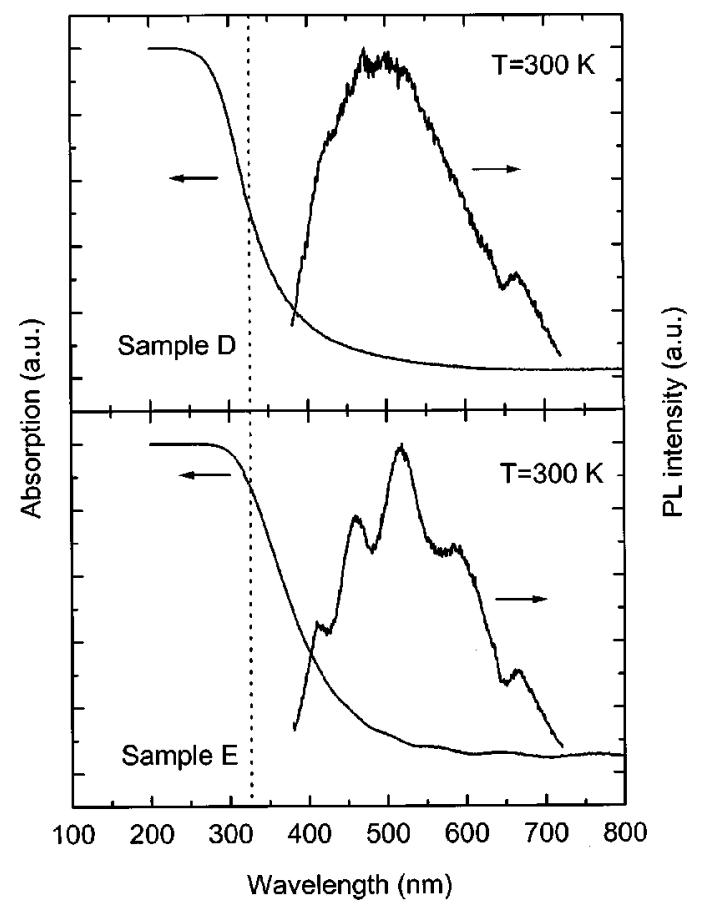

FIG. 2. Room-temperature optical absorption and steady-state PL spectra of samples D and E.

The large difference between the possible direct band gaps of samples D and E shows clear evidence for quantum confinement. However, the emission spectra of samples $D$ and $E$ exhibit independence of the cluster size, as shown in Fig. 2. Indeed, this is a quite puzzling problem, which also occurs in $\mathrm{Si}$ nanocrystals. ${ }^{20}$ Like $\mathrm{Si}$ nanocrystals, all $\mathrm{SiC}$ nanocrystals display broad emission spectra even covering the whole visible light region. Most of the peaks in the PL spectrum of sample E stem from optical interference between the top surface of the films and the top surface of the Si substrates. We also found that the luminescent characteristics of the samples are very stable. For example, we did not observe any degradation of the emission intensity eight months after the samples were stored in air. This characteristic is very important for practical applications of $\mathrm{SiC}$ nanocrystals in optoelectronic devices.

Figure 3 shows the time evolution of the luminescence intensity of the dominant emissions from samples D and E at room temperature. The solid lines show the biexponential fitting results to the experimental data. Two decay time constants of 166, and 772 ps were obtained for sample D, while decay time constants of 246 and 974 ps were identified for sample E. It should be noted that the decay times of about 1 ns are at least two orders of magnitude faster than that of the bound-exciton transitions of bulk $3 \mathrm{C}-\mathrm{SiC}$ at low temperature. $^{21}$ The biexponential decay implies that multilevels may be involved in radiative recombination. A more interesting phenomenon is that $\mathrm{SiC}$ nanocrystals at room temperature have comparable PL decay times with highquality bulk direct-band-gap semiconductors ${ }^{22,23}$ and their low-dimensional structures such as quantum wells ${ }^{24,25}$ and quantum dots 25,26 at low temperature. Recently, Wilcoxon and Samara $^{27}$ reported a radiative lifetime of $\sim 1 \mathrm{~ns}$ of the blue light emission of crystalline Si nanosized particles in the size of $\sim 2 \mathrm{~nm}$. They attribute the transition to direct recombination at the Brillouin-zone center. The observed intense AIP license or copyright, see http://apl.aip.org/apl/copyright.jsp 


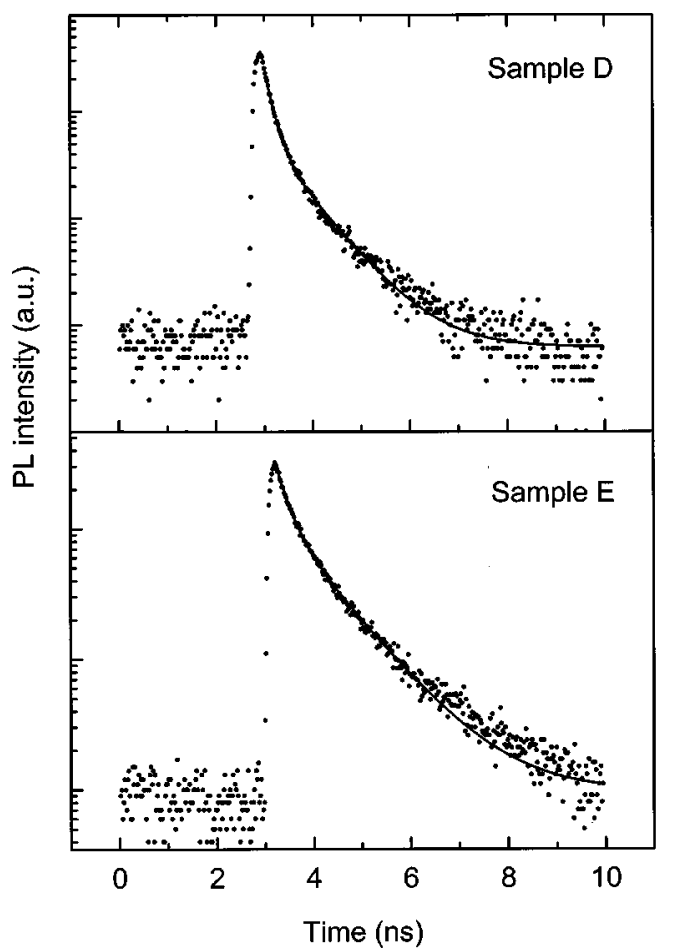

FIG. 3. Room-temperature PL decay curves of the dominant emission peaks of samples D and E. The solid circles represent the experimental data while the solid lines show the biexponential fitting results.

photon emission and 1-ns-level PL decay time strongly suggest that the dominant radiative recombinations in the $\mathrm{SiC}$ nanocrystals are from direct optical transitions. It is known that the surface states play a core role in the influence of the optical and electrical properties of nanoclusters because of such a high surface-to-volume ratio. ${ }^{28}$ Koch, Petrova-Koch, and Muschit ${ }^{29}$ suggested that surface states may act as luminescent centers in Si nanocrystals. Recently, Allan, Delerue, and Lannoo ${ }^{30}$ theoretically demonstrated the stability of the self-trapped exciton state at the surface of a Si nanocrystal using semiempirical and ab initio local-density calculations. According to their calculations, these luminescent surface states are obtained for dimer bonds passivated, for instance, by hydrogen atoms or by silicon oxide. They also found that the light emission from such trapped excitons is basically independent of cluster size. In this regard, it is noted that we use highly pure hydrogen gas as the diluting agent of $\mathrm{SiH}_{4}$ and $\mathrm{CH}_{4}$ in the synthesis. Therefore, it is quite possible that our $\mathrm{SiC}$ nanoclusters are terminated by hydrogen. Significant reduction of the nonradiative rate due to termination of dangling bonds at the nanocrystal surface was addressed previously. ${ }^{28}$ Allan et al. ${ }^{30}$ also also pointed out that the selftrapped exciton model is likely to be valid for all types of semiconductor nanocrystals. It is interesting that the lifetime of self-trapped excitons in the Si nanocluster calculated by them is close to $1 \mathrm{~ns}$. We are thus led to conjecture that the dominant optical transition in $\mathrm{SiC}$ nanocrystals is due to the surface self-trapped excitons proposed by Allan, Delerue, and Lannoo. ${ }^{30}$

In summary, we have grown nanocrystalline $\mathrm{SiC}$ films on $\mathrm{Si}$ using ECRCVD. These $\mathrm{SiC}$ films emit intense visible light at room temperature under excitation of a shortwavelength laser. The steady-state and ps time-resolved PL spectra of these samples were measured at room temperature.

Downloaded 03 Nov 2006 to 147.8.21.97. Redistribution subject
It was found that the PL decay time in the nanosecond scale is at least two orders of magnitude faster than that of the bound-exciton transitions in bulk $3 \mathrm{C}-\mathrm{SiC}$ at low temperature. Direct optical transitions, such as surface self-trapped exciton transitions, should be responsible for the dominant emissions with a decay time of $\sim 1 \mathrm{~ns}$. This nanocrystalline $\mathrm{SiC}$ film emitting strong visible light should be a promising material for fabrication of white-light LEDs.

One of the authors (S.J.X.) thanks Dr. K. Y. Chan for sharing his scanning probe microscopy equipment, and Crystal Cheung, S. H. Cao, N. Chi, and L. C. Xu for their help in AFM measurements. One of the authors (M.B.Y.) acknowledges financial support from the National Science Foundation of China.

${ }^{1}$ G. L. Harris, Properties of Silicon Carbide (INSPEC, London, U.K., 1995).

${ }^{2}$ G. Pensl, H. Morkoç, B. Monemar, and E. Janzén, Silicon Carbide, IIINitrides and Related Materials: Parts I and II (Trans. Tech., Switzerland, 1998).

${ }^{3}$ SiC Materials and Devices, Semiconductors and Semimetals Vol. 52, edited by Y. S. Park (Academic, London, U.K., 1998).

${ }^{4}$ L. Hoffman, G. Ziegler, D. Theis, and C. Weyrich, J. Appl. Phys. 53, 6962 (1982).

${ }^{5}$ D. Engemann, R. Fischer, and J. Knecht, Appl. Phys. Lett. 32, 567 (1978).

${ }^{6}$ H. Munekata and H. Kukimoto, Appl. Phys. Lett. 42, 432 (1983).

${ }^{7}$ D. Kruangam, T. Endo, W. Guang-Pu, H. Okamoto, and Y. Hamakawa, Jpn. J. Appl. Phys., Part 2 24, L806 (1985).

${ }^{8}$ D. Kruangam, in Amorphous and Microcrystalline Semiconductor Devices: Optoelectronic Devices, edited by J. Kanicki (Artech House, 1991), Chap. 6.

${ }^{9}$ S. Y. Lee, K. C. Song, J. Kim, J.-H. Son, and J. Ahn, Jpn. J. Appl. Phys., Part 1 37, 6841 (1998).

${ }^{10}$ A. R. Shimkunas, P. E. Mauger, L. P. Bourget, R. S. Post, L. Smith, R. F. Davis, G. M. Wells, F. Carrina, and R. B. McIntosh, J. Vac. Sci. Technol. B 9, 3258 (1991).

${ }^{11}$ T. Matsumoto, J. Takahashi, T. Tamaki, F. Futagi, H. Mimura, and Y. Kanemitsu, Appl. Phys. Lett. 64, 226 (1994).

${ }^{12}$ H. Mimura, T. Matsumoto, and Y. Kanemitsu, Appl. Phys. Lett. 65, 3350 (1994).

${ }^{13}$ A. O. Konstantinov, A. Henry, C. I. Harris, and E. Janzén, Appl. Phys. Lett. 66, 2250 (1995).

${ }^{14}$ L. S. Liao, X. M. Bao, Z. F. Yang, and N. B. Min, Appl. Phys. Lett. 66, 2382 (1995)

${ }^{15} \mathrm{M}$. B. Yu, Rusli and S. F. Yoon (unpublished).

${ }^{16} \mathrm{~S}$. Yoshida, in Properties of Silicon Carbide, edited by G. L. Harries (INSPEC, London, U.K., 1995), p. 76.

${ }^{17}$ K. Järrendahl and R. F. Davis, in SiC Materials and Devices, Semiconductors and Semimetals, Vol. 52, edited by Y. S. Park (Academic, London, U.K., 1998), p. 14.

${ }^{18}$ R. J. Elliot, Phys. Rev. 106, 1384 (1957).

${ }^{19}$ M. Ben-Chorin, B. Averboukh, D. Kovalev, G. Polisski, and F. Koch, Phys. Rev. Lett. 77, 763 (1996).

${ }^{20}$ D. J. Lockwood, Solid State Commun. 92, 101 (1994).

${ }^{21}$ J. P. Bergman, E. Janzén, S. G. Sridhara, and W. Choyke, Silicon Crabide, III-Nitrides and Related Materials: Part I (Trans. Tech., Switzerland, 1998), p. 485.

${ }^{22}$ G. W. 't Hooft, W. A. J. A. van der Poel, and L. W. Molenkamp, Phys. Rev. B 35, 8281 (1987).

${ }^{23}$ O. Brandt, J. Ringling, K. H. Ploog, H.-J. Wünsche, and F. Henneberger, Phys. Rev. B 58, R15977 (1998).

${ }^{24}$ J. Feldmann, G. Peter, E. O. Göbel, P. Dawson, K. Moore, C. Foxon, and R. J. Elliott, Phys. Rev. Lett. 59, 2337 (1987).

${ }^{25} \mathrm{P}$. Lefebvre, J. Allègre, B. Gil, A. Kavokine, H. Mathieu, W. Kim, A. Salvador, A. Botchkarev, and H. Morkoç, Phys. Rev. B 57, R9447 (1998).

${ }^{26}$ M. Colocci, A. Vinattieri, L. Lippi, F. Bogani, M. Rosa-Clot, S. Taddei, A. Bosacchi, S. Franchi, and P. Frigeri, Appl. Phys. Lett. 74, 564 (1999).

${ }^{27}$ J. P. Wilcoxon and G. A. Samara, Appl. Phys. Lett. 74, 3164 (1999).

${ }^{28}$ See, for example, A. P. Alivisatos, Science 271, 933 (1996).

${ }^{29}$ F. Koch, V. Petrova-Koch, and T. Muschit, J. Lumin. 57, 271 (1993).

${ }^{30} \mathrm{G}$. Allan, C. Delerue, and M. Lannoo, Phys. Rev. Lett. 76, 2961 (1996).
AIP license or copyright, see http://apl.aip.org/apl/copyright.jsp 